\title{
Theranostic Applications of Neurotensin
}

\section{Alan D Goddard*}

School of Life and Health Sciences, University of Aston, Birmingham, UK, B4 7ET

Date of Receipt- 07/04/2017
Date of Revision- 15/04/2017
Date of Acceptance- 23/04/2017
Address for
Correspondence
School of Life and Health
Sciences, University of Aston,
Birmingham, UK, B4 7ET
E-mail:
a.goddard@aston.ac.uk

\begin{abstract}
Targeted theranostics is increasingly attractive in the cancer field to reduce off-target effects and provide rapid diagnosis. Neurotensin (NT) and its derivatives target neuortensin receptor 1 (NTS1) which has strong, selective, aberrant expression in certain tumour cells. This commentary briefly describes the advantages of targeting using the NT/NTS1 pairing and discusses a recent article on Theranostic Value of Multimers: Lessons Learned from Trimerization of Neurotensin Receptor Ligands and Other Targeting Vectors that questions the relevance of in vitro studies with these theranostics to in vivo applications. This remains an area of great interest and the rational design of targeting molecules with high in vivo efficacy is a key goal of the field.
\end{abstract}

Keywords: Targeted drug delivery, Neurotensin.

\section{INTRODUCTION}

Targeting molecular changes on the surface of cancer cells is desirable as a route to reduce off-target effects and dose reduction ${ }^{1}$. These biomarkers can often be targeted for drug delivery and rapid diagnosis (theranostics). A range of targeting options are available but it is becoming increasingly common to utilise peptides that target cell-surface receptors that are aberrantly expressed in cancer ${ }^{2}$. Neurotensin is a tridecapeptide with key physiological roles in neuromodulation and as a local peripheral hormone ${ }^{3}$. Its actions are mediated by two $\mathrm{G}$ protein-coupled receptors, neurotensin receptor 1 (NTS1) and NTS2, along with NTS3/sortilin. Interestingly, NTS1 has been demonstrated to be upregulated in a variety of tumours including those in the breast and pancreas, tissues in which it is not natively expressed ${ }^{4}$. These findings have led to the investigation of the NT/NTS1 axis as a tumour targeting mechanism.

The upregulation of NTS1 on cancerous cells makes it attractive both for therapeutics and diagnostics and a number of approaches have been taken ${ }^{5-9}$. The minimal receptor-binding element of NT is the last six amino acids (NT8-13) which binds NTS1 with subnanomolar affinity ${ }^{10}$. However, NT and its derivatives are unstable in plasma having half-lives measured in minutes ${ }^{11}$. As such, stabilised peptides have been developed which retain high-affinity binding to NTS1 whilst having dramatically increased half-lives ${ }^{12}$. Upon ligand (NT) binding, NTS1 is internalised along with the ligand and there is evidence that NT escapes the lysosome and subsequent degradation ${ }^{13}$. Therefore, NT-conjugated therapeutics has potential to target and kill cancerous cells.

A number of studies have focussed on modification of NT for theranostics but an area of increasing interest is the production of multimers of NT. These multimers have higher affinity for receptors than monomers, presumably due to their ability to interact with multiple neighbouring receptors. These oligobranched or multimeric peptides have been used to deliver liposomal therapies to cells as well as for targeting of small molecules have used ${ }^{6,7,9}$. Potential problems with such theranostics include stability, especially of the NT portion, and off-target effects.

A recent study used gallium-labelled trimeric metabolically-stabilised NT (NT4). This compound was generated using the TRAP (1,4,7-triazacyclononane-1,4,7-tris[(2-carboxyethyl)methylenephosphinic acid) methodology which simplifies oligomerisation and facilitates efficient radioactive labelling with ${ }^{68} \mathrm{Ga}$. The peptide retained the sub-nanomolar affinity which makes NT such an attractive targeting molecule for use in cancer diagnostics and treatment ${ }^{14}$. 
Whilst this study showed that the $\left[{ }^{68} \mathrm{Ga}\right]$ Ga-TRAP(NT4) ${ }_{3}$ peptide was rapidly and effectively internalised into the HT29 colorectal adenocarcinoma cell line in vitro, efflux was relatively rapid as well ${ }^{14}$. Injection of HT29 cells into nude mice generated model tumours. Subsequent injection of $\left[{ }^{68} \mathrm{Ga}\right] \mathrm{Ga}-\mathrm{TRAP}(\mathrm{NT} 4)_{3}$ into the tail vein revealed substantial uptake into the kidneys and also the liver with a low clearance rate. It did, however, show moderate uptake into HT29 tumours that was increased compared to an equivalent monomeric peptide conjugate. Whilst the in vitro properties of the trimer were more desirable than the monomer, this did not translate into in vivo efficacy.

The study also compared the effects of other Ga-labelled timeric peptides and concluded that, whilst trimeric compounds showed increased affinity to their targets compared to the monomers, no systematic improvement of in vivo efficacy was observed ${ }^{14}$. To conclude, whilst NT (and other peptides) show promise as targeting moieties and trimerisation increases affinity, more work is needed to allow the rational design of conjugates which will demonstrate robust in vivo performance. Much recent progress has been demonstrates with NT as a theranostic and this will continue to represent a valuable avenue of research ${ }^{15}$.

\section{REFERENCES}

1. Gudkov SV, Shilyagina NY, Vodeneev VA, et al. Targeted radionuclide therapy of human tumors. Int J Mol Sci. 2016;17(1).

2. Accardo A, Aloj L, Aurilio M, et al. Receptor binding peptides for target-selective delivery of nanoparticles encapsulated drugs. Int $\mathrm{J}$ Nanomedicine. 2014;9:1537-57.

3. Mustain WC, Rychahou PG, Evers BM. The role of neurotensin in physiologic and pathologic processes. Curr Opin Endocrinol Diabetes Obes. 2011;18(1):75-82.

4. Dupouy S, Mourra N, Doan VK, et al. The potential use of the neurotensin high affinity receptor 1 as a biomarker for cancer progression and as a component of personalized medicine in selective cancers. Biochimie. 2011;93(9):1369-78.

5. Jia Y, Shi W, Zhou Z, et al. Evaluation of DOTA- chelated neurotensin analogs with spacerenhanced biological performance for neurotensinreceptor-1-positive tumor targeting. Nucl Med Biol. 2015;42(11):816-23.

6. Brunetti J, Falciani C, Lelli B, et al. Neurotensin branched peptide as a tumor-targeting agent for human bladder cancer. Biomed Res Int. $2015 ; 173507$.

7. Falciani C, Accardo A, Brunetti J, et al. Targetselective drug delivery through liposomes labeled with oligobranched neurotensin peptides. Chem Med Chem. 2011;6(4):678-85.

8. Falciani C, Brunetti J, Lelli B, et al. Nanoparticles exposing neurotensin tumor-specific drivers. $\mathrm{J}$ Pept Sci. 2013;19(4):198-204.

9. Falciani C, Brunetti J, Lelli B, et al. Cancer selectivity of tetrabranched neurotensin peptides is generated by simultaneous binding to sulfated glycosaminoglycans and protein receptors. J Med Chem. 2013;56(12):5009-18.

10. Kitabgi P. Functional domains of the subtype 1 neurotensin receptor (NTS1). Peptides. 2006;27(10):2461-68.

11. Lee YC, Uttenthal LO, Smith HA, et al. In vitro degradation of neurotensin in human plasma. Peptides. 1986;7(3):383-7.

12. Mascarin A, Valverde IE, Mindt TL. Structureactivity relationship studies of amino acid substitutions in radiolabeled neurotensin conjugates. Chem Med Chem. 2016;11(1):102-7.

13. Vandenbulcke F, Nouel D, Vincent JP, et al. Ligand-induced internalization of neurotensin in transfected COS-7 cells: differential intracellular trafficking of ligand and receptor. Journal of Cell Science. 2000;113(17):2963-75.

14. Maschauer S. Theranostic value of multimers: lessons learned from trimerization of neurotensin receptor ligands and other targeting vectors. Pharmaceuticals. 2017;10(1).

15. Maschauer S, Einsiedel J, Reich D, et al. theranostic value of multimers: lessons learned from trimerization of neurotensin receptor ligands and other targeting vectors. Pharmaceuticals. 2017;10(1):29. 